\title{
The impact of chemosensory and food-related changes in patients with advanced oesophagogastric cancer treated with capecitabine and oxaliplatin: a qualitative study
}

\author{
Yfke C. de Vries ${ }^{1,2}$ • Esther Helmich ${ }^{3,4}$ - Matty D. A. Karsten ${ }^{2}$ - Sanne Boesveldt ${ }^{2}$. \\ Renate M. Winkels ${ }^{2} \cdot$ Hanneke W. M. van Laarhoven ${ }^{5}$
}

Received: 26 November 2015 / Accepted: 9 February 2016 /Published online: 27 February 2016

(C) The Author(s) 2016. This article is published with open access at Springerlink.com

\begin{abstract}
Purpose Chemosensory changes are frequently observed side effects of cytotoxic treatment and have an impact on daily life by altering food-related behaviour and daily practices. For oesophagogastric cancer patients, these changes can be particularly important as they may have specific needs with regard to eating, due to obstruction of the upper intestinal tract. The purpose of this study was to gain insight into the impact of chemosensory and food-related changes in oesophagogastric cancer patients undergoing chemotherapy and how this may influence the practical and social aspects of food-related behaviour of patients and their relatives.

Methods We used a qualitative interview approach with a cross-sectional design using semi-structured interviews. Template analysis was used to analyse patients' experiences with and the impact of chemosensory changes on daily life. Thirteen advanced oesophagogastric cancer patients treated with capecitabine and oxaliplatin were included by
\end{abstract}

Yfke C. de Vries and Esther Helmich share first authorship.

Yfke C. de Vries

yfke.devries@wur.nl

1 Top Institute Food and Nutrition, P.O. Box 557, Wageningen 6700 AN, The Netherlands

2 Division of Human Nutrition, Wageningen University, P.O. Box 8129, 6700 EV Wageningen, The Netherlands

3 Center for Evidence-Based Education, Academic Medical Center, Amsterdam, The Netherlands

4 Rehabilitation and Care Center Vreugdehof, Amsterdam, The Netherlands

5 Department of Medical Oncology, Academic Medical Center, Amsterdam, The Netherlands convenience sampling, recruited from one academic hospital, and interviewed at home or in the hospital.

Results There was a large variation in the impact of chemosensory changes in oesophagogastric cancer (OGC) patients, though daily life was impacted substantially when chemosensory and/or food-related changes were experienced. Three main themes emerged from the interviews: altered food preferences, practical constraints in daily life, and impact on social functioning.

Conclusion Chemosensory and food-related changes significantly influenced food preferences and had practical and social consequences in daily life of patients and their relatives. Specific nutritional care for these patients should be directed towards enhancing food enjoyment and should take the specific needs, related to the location of the tumour, into account.

Keywords Cancer $\cdot$ Chemotherapy $\cdot$ Taste $\cdot$ Smell $\cdot$ Food preferences

\section{Introduction}

Taste and smell alterations are among the most common side effects in cancer patients undergoing cytotoxic treatment [1-3]. Studies have reported a prevalence of 45 to $84 \%$ for self-reported taste changes and 5 to $60 \%$ for smell changes among cancer patients undergoing chemotherapy [2]. These changes can lead to a decreased appetite, liking and enjoyment of food, food avoidance, altered food preferences, food aversions, a reduction in food intake and eventually malnutrition in cancer patients [1, 4-10]. These different alterations in taste and smell may seriously impact patients' daily life and quality of life [11-13]. For instance, taste and smell changes can affect daily living, change practical routines and may also have an impact on social functioning related to cooking and rituals 
of eating, such as eating together with family and friends [11, $14,15]$. In order to support patients in the course of their anticancer treatment, it is important to further explore these experienced chemosensory changes.

To date, most studies assessing chemosensory changes during chemotherapy were executed in heterogeneous study populations undergoing a variety of chemotherapeutic regimens [2]. However, it has been suggested that the experience of taste and smell changes may depend on specific cytotoxic agents used [16]. Additionally, patients with certain cancer types may experience specific difficulties with respect to eating. For instance, in patients with oesophagogastric cancer (OGC), eating may be particularly complicated by obstruction of the upper intestinal tract, potentially resulting in a poor nutritional status [17]. Therefore, it is of specific interest to study how patients and their families experience the interactions of these complications with chemosensory alterations resulting from cytotoxic treatment.

So far, no studies have focussed on the impact and consequences of chemosensory changes within advanced OGC patients receiving chemotherapy. In order to improve supportive care, in particular nutritional advice and management strategies for OGC patients with chemosensory changes, a better understanding of the experiences of patients is needed. Not only the experience of chemosensory changes and dietary itself are of interest but also the impact of these experiences and the consequences of these changes in daily life.

In this study, we use a qualitative approach to explore the impact of chemosensory and food-related changes in advanced oesophagogastric cancer patients undergoing chemotherapy with two specific cytotoxic agents, capecitabine and oxaliplatin, and study how this influences the practical and social aspects of food-related behaviour of patients and their relatives.

\section{Methods}

\section{Study design}

We adopted qualitative approach, applying template analysis to describe and interpret the lived experience of patients in order to get a deep understanding of these experiences at both a general and individual level [18]. The study was exempt from formal ethical approval by the institutional medical ethics committee of the Academic Medical Centre (W14_010).

\section{Participants}

We included a convenience sample of patients with OGC diagnosed in the Academic Medical Centre (AMC) in Amsterdam, the Netherlands. The following inclusion criteria were applied: patients with diagnosed oesophageal and stomach cancer who had a metastatic or unresectable carcinoma at the time of diagnosis with a WHO performance status of 0 to 2 [19], who were currently receiving palliative chemotherapy with capecitabine $\left(\right.$ Xeloda $\left.^{\circledR}\right)$ and oxaliplatin $\left(\right.$ Eloxatin $\left.^{\circledR}\right)$ (CAPOX) and had completed at least two cycles of chemotherapy. We considered convenience sampling to be adequate for broadly exploring the experiences of chemosensory changes following chemotherapy. Patients were not purposefully selected on reporting an altered chemosensory perception.

\section{Data collection}

MK, a clinical dietician and MSc student Nutrition and Health at the time of the study, carried out semi-structured interviews. She had no prior relationship with the participants. Interviews were held at home or in the hospital, following patients' preference, and lasted approximately 15-60 min. In the hospital, rooms could be private or shared with other patients. During the interviews, patients were often accompanied by a close relative or friend, who was allowed to participate in the conversation. The interview guide was developed based on key topics from literature and investigators' knowledge and experiences from clinical practice and further adapted in the course of the study. Interviews covered changes in taste and smell, appetite, enjoyment of food, food preferences, practical and social consequences, strategies to handle changes and the impact of changes in smell, taste on daily life and the impact of such changes upon diagnosis. Interviews were audio recorded.

\section{Data analysis}

Interviews were transcribed and coded according to the template analysis as described by King [20]. Template analysis is a thematic analysis where, to analyse the data, the researcher identifies a number of codes or themes that summarize key ideas, concepts, actions or experiences extracted from the interviews by reading and re-reading the text. Codes are organized hierarchically with the highest level codes representing broad themes in the data and lower-level codes representing more narrow or specified themes in the data. When patients did not experience any changes in chemosensory function, interviews were not or only partly transcribed, although all interviews were reviewed for the final template.

The first two interviews were coded for main themes by $\mathrm{MK}$, together with and $\mathrm{YV}$, a $\mathrm{PhD}$ student in the field of sensory science and eating behaviour and studies chemosensory changes during chemotherapy in cancer patients. The template was further developed in discussion with $\mathrm{EH}$, an elderly care physician with specific expertise in qualitative research, who was working on separate transcripts. MK and YV constructed the final template on the basis of detailed re-reading of the full set of transcripts and discussed their 
interpretations with EH and HvL, a medical oncologist specialized in the treatment of OGC patients.

\section{Results}

Thirteen patients were interviewed for this study, of whom demographic and diagnostic data are summarized in Table 1. We defined nine themes, each divided into further sub-themes (Table 2). Three main themes pertained strongly to the experience of patients and are fully described: altered food preferences, practical constraints in daily life and the meaning of chemosensory and food-related changes for social life.

\section{General findings}

We found a large individual variation in the perceived degree and impact of chemosensory changes and other side effects of chemotherapy in patients. Patients who experienced mild or few side effects mostly did not experience alterations in their sense of smell or taste either. In contrast to other side effects of chemotherapy, patients did not always mention changes in taste and smell spontaneously. Nevertheless, these changes were described as important or as having a substantial impact on their daily lives. Some patients reported that their daily lives were greatly impacted by chemosensory changes, while others experienced the impact as less significant. Two extremes are mentioned; Sam, a patient with only minor chemosensory complaints, said:

I do find them noticeable [changes in taste and smell], but I think it's more important to live than to hand in some of the sensory stimuli. (Sam)

Charles, a patient with severe complaints, believes that these chemosensory changes do not outweigh the benefits of chemotherapy.

I would not do it again [chemotherapy] if I knew what I know now. (...) Should I be severely ill for nine or ten weeks for just a few months extension of my life? (Charles)

Patients found it difficult to describe changes in taste and smell perception as distinct features and referred to changes in flavour as a whole or instead jointly ascribed these to changes in taste perception. They were, however, perfectly able to articulate what these changes entailed:

I used to love cheese, but if I eat cheese now, I cannot taste it. I only taste the saltiness. I also used to love quark with blueberries.(...) If you would close your eyes and take a bite it just doesn't taste the way that it used to do. (Nicole)

Patients described changes in taste or smell in terms of having a reduced enjoyment of food.

You just hope that it's over soon, because you don't enjoy food whatsoever. You just eat because you have to, but there is no pleasure in it. You just hope that it will improve soon. (Jacob)

\section{Altered food preferences}

A variety of altered food preferences were experienced by patients, which are expressed by a need for more or less intense flavours, changed food choices and food aversions.

To compensate for altered chemosensory perception, some patients described a need for more intense flavours, which was reflected by addition of condiments, like sugar, seasonings and salt or by choosing products with a distinctive flavour.

I recognised, that during the period that I started tasting less, I used more salt and more products with strong flavours. I had also figured to eat herring: besides a lot of nutrients, it also has a strong flavour. (Abraham)

Altered chemosensory perception did not always result in a preference for more intense stimuli; in contrast, some patients described a need for less intense flavours, specifically for spicy products.

I need to be a bit careful not to eat spicy foods. Last Wednesday for instance, I made beans with a spicy curry paste and I had to pay the bill all night. That was too spicy, so I need to take into account not to use too much spicy herbs. (Nicole)

Besides preferences for more or less intense flavours, patients sometimes needed to choose other types of food. Chemotherapy induced an enhanced sensitivity to cold, particularly a few days after oxaliplatin infusion, which resulted in a preference for warm foods, the need to wear gloves to get food out of the refrigerator or an urge to put all foods in the microwave before consumption.

Many patients talked about eating food that could easily pass the oesophagus. Some patients needed to switch to liquid foods, in order to combat weight loss and maintain sufficient energy intake. Some specific dry foods, like bread and meat were avoided, because it could not easily pass the oesophagus.

It should easily slide through, it should not be too dry and it has to be tasteful. (Sam) 


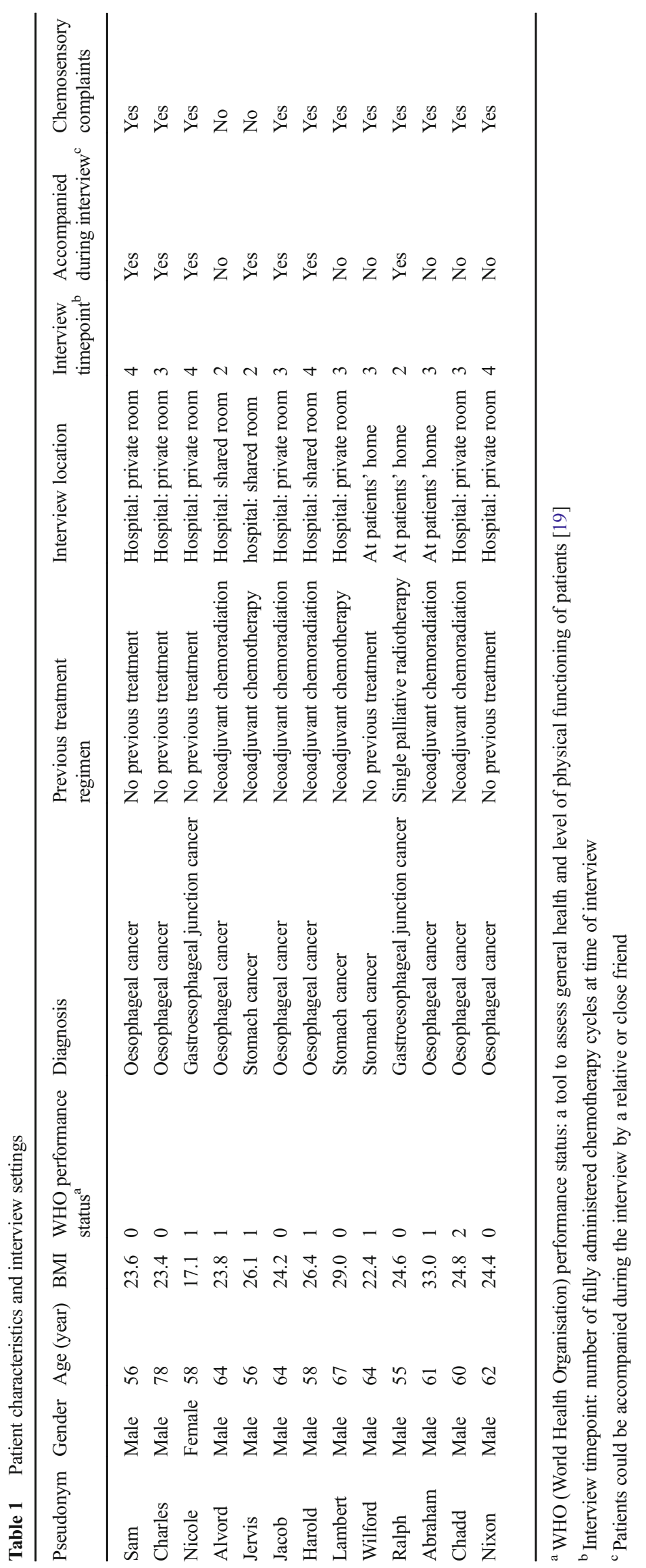


Table 2 Template

1. Changes in taste

1.1. Reduced taste perception

1.2. Enhanced taste perception

1.3. Altered taste perception

1.4. Constant taste without the presence of food (phantom)

2. Changes in smell

2.1. Reduced odour perception

2.2. Enhanced odour perception

2.3. Altered odour perception

3. Appetite

3.1. Decreased appetite

3.2. Improved appetite

4. Nutritional advice (dietician)

4.1. Adding more fat to the diet/eating products that contain more fat

4.2. Eating more protein-rich foods

4.3. Eating more frequently

5. Altered food preferences

5.1. Need for a more intense flavour

5.1.1. Addition of condiments

5.1.2. Choose products with a more distinctive flavour

5.2. Need for a less intense flavour

5.2.1. Less addition of seasonings

5.3. Changed food choices

5.3.1. Favouring warm food

5.3.2. Taking into account patients' food preferences

5.3.3. Food for easy passage oesophagus

5.3.3.1. Liquid and smooth food

5.3.3.2. Avoiding dry and grainy food

5.3.3.3. Drink while eating

5.4. Food aversions

5.4.1. Decreased enjoyment in eating food

5.4.1.1. Not liking anything anymore

5.4.1.2. Counting nutrients

5.4.2. Aversion to specific foods

5.4.2.1. Aversion towards fried food and hot meals

5.4.2.2. Aversion towards meat

6. Practical constraints in daily life

6.1. Not being able to eat/drink

6.1.1. Not being able to eat/drink cold products

6.2. Not being able to swallow the food

6.3. Planning meals and dinners

6.4. Adapting to what is still possible to eat

6.4.1. Liquid and smooth food

6.4.2. Avoiding dry and grainy food

6.4.3. Drink while eating

6.4.4. Trying different foods

6.4.5. Eating more frequently

6.4.6. Eating less (frequently)

6.4.7. Eating smaller portions

7. Social functioning in daily life
Table 2 (continued)

7.1. Eating (together) less sociable

7.1.1. Fewer dinners with family and friends

7.1.2. Planning of meals

7.2. Role of partners and family

7.2.1. Taking into account patients' food preferences

7.2.2. Family is motivating (forcing?) patient to eat

7.2.3. Change of family roles

7.3. Decreased pleasure in eating

8. Significance of chemosensory changes upon diagnosis

8.1. Limited impact of chemosensory changes compared to

chemotherapy benefits

8.2. Major impact of chemosensory changes compared to

chemotherapy benefits

9. General side effects of chemotherapy

9.1. Food-related side effects

9.2. Other side effects

10. Changes over time

10.1. Exacerbation of side effects with increased number of chemotherapy cycles

10.2. Higher intensity of side effects at start of chemotherapy

Another way to swallow dry foods was to drink along with food. For Wilford this was a way to force the food to go down.

If I do not manage to get the food down, I take a glass of apple juice, and swallow the food along with the apple juice just to get it inside. I just have to get it inside. (Wilford)

Patients often complained about food aversions, which were frequently related to aversive cooking aromas. These aversions could be directed at specific foods, like meat, or more general towards reduced enjoyment of eating.

If you cook vegetarian, or just normal, it has a certain smell. (...) It gives a smell which I find really annoying now. It causes me to lose my appetite before eating it (Wilford)

Changed food preferences were a challenge for partners and relatives, who had to take the altered food preferences into account during shopping and cooking.

You make sure that when you cook a meal you know that he likes it (...) I used to make him some oven baked fish,. .. but as soon as it was time to eat he looked at his plate, took two bites and said "That's enough, well you don't have to cook that for me anymore." (Charles's wife)

\section{Practical constraints in daily life}

Patients encountered many practical constraints in daily life, mostly because of obstruction of the oesophagus. These 
pertained to not being able to eat, drink or swallow, consequences for the planning of meals and dinners, and adapting to what was still possible to eat.

For some patients the daily routine was changed, meals and dinners were planned at different times of the day. For instance for Chadd, the daily pattern was changed in order not to get any gastric refluxes during the night.

I'll eat as much as possible preferably in the early afternoon, around $2 \mathrm{pm}$ or so. Not in the evening around 5 or $6 \mathrm{pm}$. Because I've had some of these refluxes at night, it was complete panic. (Chadd)

Not being able to swallow the foods made patients slow down and take their time while consuming a meal and therefore taking much more time.

It seems as if the food does not pass that fast, as if it gets stuck. You have to slow down your eating, take pauses between bites. (Nicole)

Patients had to adapt to what was still possible to eat, not only by choosing or avoiding specific types of food as previously described but also by changing their food pattern into eating smaller portions, eating less frequently, or more frequently but in smaller portions.

I rather eat more frequent during the day and smaller portions than once or twice a large meal. (Sam)

\section{Social functioning in daily life}

Changes in social functioning were presented in several ways; patients felt restricted because eating was less sociable, needed to be planned and was less pleasurable. Furthermore, partners and family members played a role in social functioning by adjusting themselves to the altered food preferences which sometimes resulted in a change of roles within the family.

Eating with family and friends was less sociable because it was less spontaneous and needed to be planned. Furthermore, patients sometimes avoided going out for dinner from a fear of not having the appropriate choices on a menu, or a fear for complaints during eating.

But if you would say: 'let's go out for dinner this week with the four of us', I would say: rather not, I'm not that good accompany. (Nixon)

Partners played an important role in the social aspect of eating. Relatives often tried to stimulate patients to eat, by taking into account patients' food preferences and ensuring sufficient nutritional intake, which made mealtime less spontaneous.

We try to do that [making decisions about dinner] together. Beforehand we think about meals for today or for the rest of the week. And by doing so, she [wife] takes care that it is not too dry and so on. (Sam)

Patients sometimes tried to force themselves to eat from a feeling of guilt towards their partners because of their efforts into making food tasty.

You are eating and think: I'll stuff it inside, but for my wife it's not pleasant at all because she is trying to make something out of it. I'll stuff it inside, but actually for me it didn't have to, because it just doesn't taste right. (Jacob)

The social aspect of cooking and eating was also changed by a switch of roles for patients and partners in the household. A partner may take over grocery shopping and cooking, because a patient was not able to do it anymore, or to keep a patient from losing appetite from cooking smells by cooking $\mathrm{him} /$ herself.

Chemosensory changes influenced social aspects of eating resulting in a decreased pleasure in eating, where the role of a meal changed from something pleasurable into a compulsory way to ingest enough nutrients.

Nowadays eating is a 'necessary evil'. So I do think the social aspect is noticeable. You are counting the protein and nutrients and it feels like a mathematical exercise. My wife is thinking about the meals we shall try this time. It is a quest to find what is possible and what tastes good and so on. Nutrition and taste have a whole new impact in that way, it's noticeable and not for the better. (Sam)

\section{Discussion}

This study provides insight on the impact of chemosensory and food-related changes on the life's of oesophagogastric cancer patients undergoing palliative chemotherapy. We found a large individual variation in the intensity and impact of changes in taste or smell perception among patients. When patients experienced chemosensory and/or food-related changes, this did not only result in altered food preferences but also had practical implications and meaningfully influenced social life. 
In the current study, a relatively homogeneous group was interviewed, undergoing the same cytotoxic treatment, while other studies that examined chemosensory changes mainly used heterogeneous groups with respect to type of cancer and/or treatment [2]. We found that, also in a specific, rather homogeneous group, the impact and meaning of chemosensory changes greatly vary. The experiences of chemosensory changes found in the current study generally support existing literature on this topic with respect to altered food preferences and changed social aspects of eating. More specific for this particular group of patients with OGC are the preferences and practical constraints regarding eating food that would easily pass the oesophagus.

Food preferences were influenced by chemosensory changes during chemotherapy in several ways. These are in concordance with previous studies where a need for both more and less intense flavours and food aversions to cooking aromas were described [1,2, 21]. Typical for oxaliplatin treatment was the increased sensitivity to cold food, which resulted in a preference for warm food [22].

Although it seems an obvious observation that the obstruction of the oesophagus has consequences for food preferences and dietary patterns, there is actually little literature available on the experiences of this phenomenon in this patient group. Studies in OGC patients have mainly focussed on experiences after surgery rather than during or after chemotherapy [23, 24]. To our knowledge, this is the first study specifically focussing on the experience with regard to eating in this patient group.

Changes in social aspects of eating were reported as burdensome and entailed several aspects of social functioning. Similar to previous studies, mealtime acquired a new meaning by becoming a forced way to ingest nutrients, rather than an enjoyable part of the day [22, 23, $25,26]$. Social consequences, like the inability to eat with family and friends and altered family roles, have been reported in previous studies among patients with various cancer sites undergoing chemotherapy [11, 22]. However, changed social functioning may not be solely due to chemosensory changes, as these social consequences have been reported in studies in oesophageal cancer patients that do not undergo chemotherapy, as well [23, 25].

Oesophagogastric cancer patients are susceptible to weight loss, which is associated with a reduced quality of life and a poor prognosis of the disease [17]. The current study shows that OGC patients undergoing chemotherapy both have problems regarding chemosensory changes and difficulties with obstruction of the oesophagus, hence making nutritional advice more complex. Nutritional advice for this patient group therefore should be multidimensional and go beyond aiming for sufficient protein and energy intake, mainly taking into account the changed food preferences and practical constraints, and should aim towards enhancing the enjoyment of food. Furthermore, care strategies should not only be directed at the patient but should also take into account the role that the partner and family play in a household. Finally, health-care professionals should explicitly ask for chemosensory changes, as patients do not always mention these side effects spontaneously. Nevertheless, the results of our study show that these changes can have a substantial impact on daily life and therefore should not be disregarded in hospital practice.

Applying template analysis as a method to explore the experiences of patients with OGC resulted in a rich description of the impact of chemosensory changes and altered food preferences on the daily life of patients and their relatives. The inclusion of a small, homogenous sample of study participants allowed for in-depth exploration of the lived experience of having OGC and suffering from chemosensory changes. We did not specifically select patients with previously reported taste or smell alterations, which has been done mostly in previous qualitative studies investigating chemosensory changes during chemotherapy [11, 22, 27], but instead included also patients experiencing no or little changes in their taste and smell perception, to gain insight into a broad range of experiences. However, including only patients from the Netherlands, where standard cytotoxic treatment for oesophagogastric cancer patients only includes oxaliplatin and capecitabine, may limit the transferability of our results to other treatment regimens, contexts or countries. In other cultures, pre-existing food preferences may be different from those in the Netherlands, including the addition of other spices of the use of other ways to prepare food. Moreover, food-related behaviours and the social rituals around sharing food together may differ across cultures, resulting in different practical or social consequences of both the anatomical changes related to tumour growth and the chemosensory changes following palliative chemotherapy. Future research thus should include patients from different cultural backgrounds in different contexts.

\section{Conclusion}

The present study shows that there was a large variation in the impact of chemosensory and food-related changes in OGC patients. These changes had a substantial impact on food preferences and had various practical and social consequences in the daily life of patients and their relatives. Specific nutritional care for these patients 
should be directed towards higher food enjoyment and take the specific complaints due to the location of the tumour into account.

Acknowledgments We thank the participants for their time to be interviewed in the study. Furthermore, we thank Dieuwerke Bolhuis for her advice in the start-up phase of the study and the staff of the outpatient clinical of the Department of Medical Oncology of the Amsterdam Medical Centre for their practical help.

Compliance with ethical standards The study was exempt from formal ethical approval by the institutional medical ethics committee of the Academic Medical Centre (W14_010)

Funding The project is funded by TI Food and Nutrition, a publicprivate partnership on precompetitive research in food and nutrition. The public partners are responsible for the study design, data collection and analysis, decision to publish and preparation of the manuscript. The private partners have contributed to the project through regular discussion.

Conflict of interest The authors declare that they have no conflict of interest.

Open Access This article is distributed under the terms of the Creative Commons Attribution-NonCommercial 4.0 International License (http:// creativecommons.org/licenses/by-nc/4.0/), which permits any noncommercial use, distribution, and reproduction in any medium, provided you give appropriate credit to the original author(s) and the source, provide a link to the Creative Commons license, and indicate if changes were made.

\section{References}

1. Hong JH, Omur-Ozbek P, Stanek BT, Dietrich AM, Duncan SE, Lee YW, Lesser G (2009) Taste and odor abnormalities in cancer patients. J Support Oncol 7:58-65

2. Gamper EM, Zabernigg A, Wintner LM, Giesinger JM, Oberguggenberger A, Kemmler G, Sperner-Unterweger B, Holzner B (2012) Coming to your senses: detecting taste and smell alterations in chemotherapy patients. A Systematic Review J Pain Symptom Manage 44:880-895

3. Foltz AT, Gaines G, Gullatte M (1996) Recalled side effects and self-care actions of patients receiving inpatient chemotherapy. Oncol Nurs Forum 23:679-683

4. Sherry VW (2002) Taste alterations among patients with cancer. Clin J Oncol Nurs 6:73-77

5. Boltong A, Keast R (2012) The influence of chemotherapy on taste perception and food hedonics: a systematic review. Cancer Treat Rev 38:152-163

6. Trant AS, Serin J, Douglass HO (1982) Is taste related to anorexia in cancer patients? Am J Clin Nutr 36:45-58

7. Comeau TB, Epstein JB, Migas C (2001) Taste and smell dysfunction in patients receiving chemotherapy: a review of current knowledge. Support Care Cancer 9:575-580
8. Ravasco P (2005) Aspects of taste and compliance in patients with cancer. Eur J Oncol Nurs 9(Suppl 2):S84-S91

9. Schiffman SS, Sattely-Miller EA, Taylor EL, Graham BG, Landerman LR, Zervakis J, Campagna LK, Cohen HJ, Blackwell S, Garst JL (2007) Combination of flavor enhancement and chemosensory education improves nutritional status in older cancer patients. J Nutr Health Aging 11:439-454

10. Holmes S (1993) Food avoidance in patients undergoing cancer chemotherapy. Support Care Cancer 1:326-330

11. Bernhardson BM, Tishelman C, Rutqvist LE (2007) Chemosensory changes experienced by patients undergoing cancer chemotherapy: a qualitative interview study. J Pain Symptom Manag 34:403-412

12. Duffy VB, Fast K, Lucchina LA, Bartoshuk LM (2002) Oral sensation and cancer. Lippincott Williams \& Wilkins, Philadelphia, PA

13. Epstein JB, Robertson M, Emerton S, Phillips N, Stevenson-Moore P (2001) Quality of life and oral function in patients treated with radiation therapy for head and neck cancer. Head Neck 23:389-398

14. Bernhardson BM, Tishelman C, Rutqvist LE (2009) Taste and smell changes in patients receiving cancer chemotherapy: distress, impact on daily life, and self-care strategies. Cancer Nurs 32:45-54

15. Rehwaldt M, Wickham R, Purl S, Tariman J, Blendowski C, Shott S, Lappe M (2009) Self-care strategies to cope with taste changes after chemotherapy. Oncol Nurs Forum 36:E47-E56

16. Zabernigg A, Gamper EM, Giesinger JM, Rumpold G, Kemmler G, Gattringer K, Sperner-Unterweger B, Holzner B (2010) Taste alterations in cancer patients receiving chemotherapy: a neglected side effect? Oncologist 15:913-920

17. Bozzetti F (2010) Nutritional support in patients with oesophageal cancer. Support Care Cancer 18:S41-S50

18. Brooks J, King N (2012) Qualitative psychology in the real world: the utility of template analysis 2012 British Psychological Society Annual Conference 18th - 20th April 2012 London, UK

19. Oken MM, Creech RH, Tormey DC, Horton J, Davis TE, McFadden ET, Carbone PP (1982) Toxicity and response criteria of the Eastern Cooperative Oncology Group. Am J Clin Oncol 5: 649-655

20. King N, Horrocks C (2010) Interviews in qualitative research interviews in qualitative research. Sage London.

21. Speck RM, DeMichele A, Farrar JT, Hennessy S, Mao JJ, Stineman MG, Barg FK (2013) Taste alteration in breast cancer patients treated with taxane chemotherapy: experience, effect, and coping strategies. Support Care Cancer 21:549-555

22. Boltong A, Keast R, Aranda S (2012) Experiences and consequences of altered taste, flavour and food hedonics during chemotherapy treatment. Support Care Cancer 20:2765-2774

23. Wainwright D, Donovan JL, Kavadas V, Cramer H, Blazeby JM (2007) Remapping the Body: Learning to Eat again after Surgery for esophageal cancer. Qual Health Res 17:759-771

24. Carey S, Laws R, Ferrie S, Young J, Allman-farinelli M (2013) Struggling with food and eating - life after major upper gastrointestinal surgery. Support Care Cancer 21:2749-2757

25. Watt E, Whyte F (2003) The experience of dysphagia and its effect on the quality of life of patients with oesophageal cancer. Eur J Cancer Care (Engl) 12:183-193

26. Andreassen S, Randers I, Näslund E, Stockeld D, Mattiasson AC (2006) Patients' experiences of living with oesophageal cancer. J Clin Nurs 15:685-695

27. Bernhardson BM, Olson K, Baracos VE, Wismer WV (2012) Reframing eating during chemotherapy in cancer patients with chemosensory alterations. Eur J Oncol Nurs 16:483-490 